\title{
Community-Engineering
}

\section{Systematischer Aufbau und Betrieb Virtueller Communitys im Gesundheitswesen}

Die Autoren
Jan Marco Leimeister
Helmut Krcmar
Dr. Jan Marco Leimeister
Prof. Dr. Helmut Krcmar
Technische Universität München
Wirtschaftsinformatik (I 17)
Boltzmannstr. 3
85748 Garching b. München
089 289-19532
\{leimeister, krcmar\}@in.tum.de
http://www.winfobase.de

\section{Einleitung}

Virtuelle Communitys ${ }^{1}$ (VCs) haben sich mit dem Aufstieg des Internets entwickelt und ermöglichen Austausch mit Menschen in einer Gemeinschaft. Im Unterschied zu anderen Internetangeboten ist die direkte Interaktion zwischen den Mitgliedern eines ihrer konstituierenden Elemente und sog. nutzergenerierter Inhalt macht einen signifikanten Anteil am gesamten Inhalt des Angebotes aus. Obwohl VCs schon seit geraumer Zeit Gegenstand der Forschung sind [vgl. bspw. Figa98; HaAr97; Kim99; Pree00; Rhei93; SmKo99], muss festgestellt werden, dass die theoretische Fundierung und der wissenschaftliche Kenntnisstand $\mathrm{zu}$ VCs sehr heterogen und unvollständig ist. Viele Forscher negieren darüber hinaus die Möglichkeit des systematischen Aufbaus von VCs und reduzieren ihre For- schungsarbeiten auf bereits bestehende VCs.

"There is no algorithm for community. [...] There is no step-by-step recipe that can be followed that will guarantee a specific outcome." $[\mathrm{KoSm} 96,58]$.

Gesundheitsbezogene Themen sind einer der stärksten Treiber privater Internetnutzung in den USA [FoFa03]. VCs für Patienten existieren dort in großer Zahl und teilweise mit beträchtlichem Erfolg. Umso beachtlicher ist, dass VCs für $\mathrm{Pa}$ tienten in Deutschland erst teilweise und zudem sehr langsam entstehen oder wie z. B. für Krebspatienten bis vor kurzem überhaupt noch nicht existierten [DKLK01].

Die Situation, dass eine Innovation wie VCs theoretisch großen Nutzen verspricht, gleichzeitig aber noch nicht anzutreffen ist, stellt eine Herausforderung dar, der sich die meisten wissenschaftlichen Disziplinen nicht annehmen. Für die Wirtschaftsinformatik bietet sich hier ein geeignetes Anwendungsfeld: Entwicklung und Test IT-basierter Innovationen mittels Pilotierung, d. h. die systematische Entwicklung einer IT-basierten Innovation mit anschließender Einführung, Betrieb und Evaluation im realen Anwendungsfeld.

Ziel dieses Beitrags ist es, einen nachweislich erfolgreichen Weg systematischer Entwicklung, Einführung und Betrieb einer VC für Patienten im deutschen Gesundheitswesen mittels der Pilotierung einer VC für Krebspatienten darzustellen und somit einen Beitrag zur Schließung der Wissenslücke bei der Entwicklung von

\section{Kernpunkte}

Virtuelle Communitys (VCs) stiften aufgrund des orts- und zeitunabhängigen Zugangs zu Informationen und multidirektionalem Austausch mit Menschen in ähnlichen Lebenslagen sowohl für Mitglieder als auch für Betreiber einen Nutzen. In diesem Beitrag wird ein Ansatz zum systematischen Aufbau und Betrieb von VCs vorgestellt und dessen Umsetzung am Beispiel einer realen Fallstudie im Gesundheitswesen expliziert und evaluiert. Der Beitrag zeigt:

- Community-Engineering ist möglich, VCs können erfolgreich systematisch entwickelt und betrieben werden.

- Hierfür sind insbesondere domänen- und zielgruppenspezifische Besonderheiten zu berücksichtigen.

- Wichtige Erfolgsfaktoren für VCs im Gesundheitsbereich sind Stabilität und Performanz der technischen Plattform, niederschwellige Möglichkeiten des Erstkontakts mit der VC, vertrauensunterstützende Komponenten, zielgruppen- und mediengerechte Aufbereitung von Gesundheitsinformationen sowie Usability und Barrierefreiheit des Angebots. Der limitierende Faktor für einen Communitydienst ist meist nicht die Technik, sondern die organisatorische Umsetzung.

Stichworte: Virtuelle Community, Community-Engineering, Pilotierung, Patienten, Gesundheitsinformationen, Erfolgsfaktoren, Innovation, Adoption, Diffusion 
Modellen zum erfolgreichen CommunityEngineering zu leisten. Die im Beitrag dargestellte Pilotierung hat sich über zwe Jahre hinweg erstreckt ${ }^{2}$. In ihrem Verlauf wurden die bei der systematischen Entwicklung zugrunde gelegten Designelemente und Erfolgsfaktoren anhand der Akzeptanz und des Nutzens des Angebots evaluiert. Auf dieser Grundlage werden empirisch untermauerte Erkenntnisse zum systematischen Aufbau und Betrieb von VCs für Patienten im deutschen Gesundheitswesen vorgestellt.

\subsection{Virtuelle Communitys und das Gesundheitswesen}

Trotz vielseitiger Vorteile von VCs und zahlreicher Beispiele in anderen Anwendungsgebieten (bspw. im Customer Relationship Management oder im Wissensmanagement) kann im deutschen Gesundheitswesen nicht von einem weit verbreiteten und erfolgreichen Einsatz von VCs gesprochen werden.

Viele Bereiche des Gesundheitswesens ähneln anderen Wirtschafts- und Lebensbereichen, in denen informations- und kommunikationstechnische Neuerungen erfolgreich $\mathrm{zu}$ effektiven und effizienten Abläufen und oftmals zu Verbesserungen aus sozialer, technischer und ökonomischer Sicht geführt haben. Jedoch sind viele dieser Innovationen im Gesundheitssystem (noch) nicht eingeführt worden. Beispiele sind die aktuell in der Entstehung befindliche elektronische Gesundheitskarte oder die elektronische Patientenakte. Offensichtlich gibt es Besonderheiten (bspw. besondere gesetzliche Regulierungen, die Trennung zwischen Kostenträgern, Leistungserbringern und Leistungsempfängern oder das Fehlen eines freien Wettbewerbs zwischen den beteiligten Akteuren), die es nicht zulassen, dass in anderen Bereichen bereits erfolgreiche Neuerungen 1:1 ins deutsche Gesundheitswesen übertragen werden können.

\subsection{Potenziale von Virtuellen Communitys für Patienten}

Studien zeigen, dass Nutzer von VCs weniger depressiv und ängstlich sind, weniger Beschwerden haben, Probleme im Zusammenhang mit ihrer Erkrankung besser erkennen und einschätzen und somit bessere Entscheidungen treffen können, emotional und sozial besser integriert sind, ein höheres Selbstwertgefühl besitzen, weniger Schmerzen angeben und sich besser ver- sorgt fühlen [vgl. hierzu bspw. AWCR04; $\mathrm{KBCSO3]}$.

Noch stärker als bei Onlineangeboten ist die positive Wirkung von Selbsthilfegruppen auf Patienten dokumentiert [Moel96]. Jedoch ist deren Nutzung trotz der genannten Vorteile oftmals verschwindend gering. Nach Angaben der AOK BadenWürttemberg nutzen bspw. nur ca. $5 \%$ aller Krebspatienten die Angebote von Selbsthilfegruppen. Gründe hierfür liegen in der zeitlichen und örtlichen Beschränkung derartiger Angebote sowie insbesondere in der Überwindung der Hemmschwelle zur Teilnahme an derartigen Treffen. Dies verdeutlicht das Potenzial von VCs für Patienten, die einen orts- und zeitunabhängigen sowie anonymeren Zugang liefern können. Sie sind gerade für schwach besiedelte Gebiete oder für Personen mit eingeschränkter Mobilität eine sinnvolle Ergänzung zu bisher bestehenden Informationsangeboten für Patienten.

Die Aktualität von Informationen kann in VCs oftmals wesentlich höher sein als bei anderen Medien. Dies ist im Kontext gesundheitsbezogener Informationen (bspw. $\mathrm{zu}$ neuen Behandlungsansätzen, Versorgungsleistungen, etc.) aufgrund des entsprechend dringenden Interesses von $\mathrm{Pa}$ tienten ein großer Vorteil. Außerdem lässt sich in VCs häufig eine größere Offenheit der Menschen feststellen, insbesondere wenn es um lebensbedrohende Krankheiten, schwierige Situationen oder TabuThemen geht. Die Bedarfsgerechtigkeit von Informationen im Internet kann (bei entsprechend qualitätsgesicherten Inhalten) ebenfalls deutlich höher sein als bei Angeboten in anderen Medien, da der Nutzer die einzelnen Bestandteile nach seinen Wünschen einfach zusammenstellen kann.

Neben der Möglichkeit, bedarfsgerecht und anonym auf Informationen von jedem Ort zu jeder Zeit zuzugreifen, bieten VCs durch ihre Dienste multilaterale Interaktionsmöglichkeiten. Die VC ist Sende- und Rückkanal zugleich und integriert darüber hinaus eine Vielzahl unterschiedlicher Medien. Sie kann so dem unterschiedlichen Wissens- und Informationsstand des Benutzers oft besser gerecht werden.

Die Interaktion in VCs ermöglicht eine stark ausgeprägte Empathie unter den Mitgliedern sowie eine damit verbundene emotionale Integration in eine Gemeinschaft. Empathie lässt sich im Allgemeinen durch drei Eigenschaften charakterisieren [LeRu92, 234]: a) wissen, was eine andere Person fühlt, b) füblen, was eine andere Person fühlt und c) mitfüblend antworten/ agieren auf das Leid einer anderen Person.
Preece hat sowohl in Einzelfällen als auch in größer angelegten Untersuchungen Empathie in der Kommunikation zwischen den Mitgliedern nachweisen können [Pree00]. Besonders stark ausgeprägt war diese in gesundheitsbezogenen Communitys in den USA [PrGh01, 248].

\section{Ein Rahmen für Entwicklung und Betrieb Virtueller Communitys für Patienten}

Die Ursachen der häufigen Misserfolge internetbasierter Angebote generell werden häufig in unklaren Betreibermodellen, geringem Anwenderbedarf und unerfahrenem Management vermutet [SIMW01, $221 \mathrm{ff}$.$] . Virtuelle Communitys bzw. die$ Funktionen einer Communityplattform werden dabei gerne als mögliche Lösung für diese Unzulänglichkeiten geschäftlicher, öffentlicher oder privater Internetangebote genannt [LeSc01]. Aufbau, Betrieb und Weiterentwicklung von VCs bleiben jedoch nach wie vor eine Herausforderung, für die bisher kein allgemeines Rezept existiert; jeder Einzelfall muss separat betrachtet werden.

Unterschiedliche Vorgehensmodelle aus der (meist praxisorientierten) Literatur liefern in mehr oder minder strukturierter bzw. vollständiger Art und Weise Gestaltungsmöglichkeiten und Erfolgsfaktoren für die Entwicklung und den Betrieb Virtueller Communitys [vgl. zu einzelnen Ansätzen bspw. BrMW00; HaAr97; Kim99; Pree00; Schu99 oder zusammenfassend Leim05, $76 \mathrm{ff}$.$] . Die dort genannten Er-$ folgsfaktoren lassen sich zusammenfassen und in einen generischen Entwicklungsprozess von VCs einordnen: ein Community-Building und Community-Management $(\mathrm{CM} \& \mathrm{CB})$-Vorgehensmodell, bestehend aus den Phasen Analyse, Design, Implementierung und Betrieb sowie Controlling und Evolution.

Die Einführung und der Betrieb Virtueller Communitys für Patienten im deutschen Gesundheitssystem lassen sich generell als Diffusionsprozess einer Innovation in einem sozialen System beschreiben. Rogers identifiziert fünf zentrale Einflussgrößen bei der Diffusion von Innovationen [Roge95]: (1) die Innovation selbst mit ihren wahrgenommenen Eigenschaften, (2) die Kommunikationskanäle der Verbreitung, (3) die Zeit, (4) das soziale System, in welchem sich die Diffusion voll- 
zieht, sowie (5) die Bemühungen derPromotoren (Change Agents) einer Innovation. Ein Innovationsentwickler kann insbesondere an den wabrgenommenen Eigenschaften der Innovation oder an der Verbreitung der Innovation über Kommunikationskanäle und den Bemühungen der Change Agents ansetzen. Folgende Meta-Erfolgsfaktoren wirken auf die wahrgenommenen Eigenschaften einer Innovation [Roge95, 16]:

1. Relativer Vorteil: Grad der subjektiv wahrgenommenen Vorteilhaftigkeit.

2. Kompatibilität: Grad der Übereinstimmung mit den persönlichen Ideen, Werten und Bedürfnissen potenzieller Adopter.

3. Komplexität: Grad der Verständlichkeit und Überschaubarkeit.

4. Erprobbarkeit: Grad der Testmöglichkeit vor der endgültigen Adoptionsentscheidung.

5. Beobachtbarkeit: Grad der Beobachtbarkeit und Kommunizierfähigkeit der Eigenschaften.

Aus den Phasen des Community-Buildingund Community-Management-Vorgehensmodells und diesen Meta-Erfolgsfaktoren der wahrgenommenen Eigenschaften einer Innovation nach Rogers lässt sich eine Matrix aufspannen, in der Erfolgsfaktoren Virtueller Communitys eingeordnet werden können (vgl. Tabelle 1). Damit können die unterschiedlichen Handlungsanweisungen zu Aufbau, Einführung und Betrieb Virtu- eller Communitys um Erfolgsfaktoren aus der Innovationsforschung ergänzt und in ein nach Phasen iterativ abarbeitbares Format gebracht werden.

Bei der Erläuterung der Felder des Modells ist kritisch anzumerken, dass der Anspruch auf Vollständigkeit und Allgemeingültigkeit nur für die Zielgruppe Patienten erhoben werden kann, da das Phänomen Virtuelle Community im größeren Kontext nicht exakt definierbar und abgrenzbar ist und dessen Instanzen in der Praxis daher unterschiedliche Vorgehensmodelle und Aktionen für Entwicklung und Evolution erfordern. Daher erfolgt eine Beschränkung auf allgemeine und essentielle Faktoren auf höherem Abstraktionsniveau für Virtuelle Communitys.

Tabelle 1 verdeutlicht Erfolgsfaktoren des Community-Building und Community-Managements. Einige Faktoren lassen sich nicht eindeutig nur einem einzelnen Feld der Matrix zuordnen; dies wird durch die Überlappung der Faktoren über mehrere Felder verdeutlicht.

Analyse: Die Identifizierung der Problemstellungen und Ziele für eine bestimmte Zielgruppe stellt den Ausgangspunkt der Analysephase für VCs dar [Kim99, 19]. Diese Analyse der Anforderungen an die Communityplattform soll deren späteren Erfolg sicherstellen. Erfolgsfaktoren sind die Anwendung moderner Analysemethoden für die Problemstellung (bspw. ethnografische Ansätze), die Fokussierung auf eine bestimmte Gruppe potenzieller Teilnehmer sowie die Analyse potenziell angemessener Architekturen für das gesamte Community-Support-System. Die Einbeziehung potenzieller Anwender in den Prozess der Analyse soll zur Erfüllung der sozialen Erwartung durch die technische Ausgestaltung beitragen, also zur Etablierung von sociability und usability [AsTa00].

Design: Eine VC bedarf nicht nur eines technischen, sondern auch eines sozialen Rahmenwerks, welches in das tägliche Leben der Teilnehmer eingebettet ist und in einem Medium bzw. auf einer Plattform repräsentiert wird [KeJo01, 77 ff.]. Die Virtuelle Community kann dabei aktiv durch einen Designprozess in einem Medium entwickelt werden (externalization) oder passiv in einem Evolutionsprozess durch ein Medium entdeckt werden (internalization) [LeSc01]. Designaspekte sind der logische Raum hinsichtlich Syntax und Semantik (z. B. die Sprache), das Kanalsystem (z. B. die Kommunikationsmittel) sowie die organisatorische Struktur der Community (z. B. der soziale Kontext, Rechte- und Rollenkonzepte). Die Erfolgsfaktoren des Designs einer VC sind die Sicherstellung der Transparenz der Designentscheidungen, die Gewährleistung der Usability des Systemdesigns, die Sicherstellung der Nutzenpotenziale der Beteiligten sowie die Begründung der Organisation der Community.

\begin{tabular}{|c|c|c|c|c|}
\hline Erfolgsfaktor $\begin{array}{r}\text { Cy\&le- } \\
\text { Phase }\end{array}$ & Analyse & Design & Implementierung und Betrieb & Controlling und Evolution \\
\hline Relativer Vorteil & $\begin{array}{l}\text { Analyse der Problemstellun- } \\
\text { gen und Ziele der VC }\end{array}$ & $\begin{array}{l}\text { Sicherstellung der Nutzen- } \\
\text { potenziale der Teilnehmer }\end{array}$ & $\begin{array}{l}\text { Sicherung der Qualität der } \\
\text { technischen Umsetzung }\end{array}$ & $\begin{array}{l}\text { Verwirklichung der Vorteile } \\
\text { des Geschäfts- und Betreiber- } \\
\text { modells }\end{array}$ \\
\hline Kompatibilität & $\begin{array}{l}\text { Fokussierung auf eine defi- } \\
\text { nierte Gruppe von Teilneh- } \\
\text { mern }\end{array}$ & $\begin{array}{l}\text { Begründung der Organisa- } \\
\text { tion der Gemeinschaft }\end{array}$ & $\begin{array}{l}\text { Einfachheit der Nutzung/ } \\
\text { Usability } \\
\text { Umsetzung vertrauensunter- } \\
\text { stützender Komponenten }\end{array}$ & Aufbau von Vertrauen \\
\hline Komplexität & $\begin{array}{l}\text { Analyse der Systemarchitek- } \\
\text { tur der Communityplattform }\end{array}$ & $\begin{array}{l}\text { Gewährleistung der Usability } \\
\text { und Sociability des System- } \\
\text { designs (Rechte u. Rollenkon- } \\
\text { zepte, etc.) }\end{array}$ & $\begin{array}{l}\text { Einfachheit der Nut-zung/ } \\
\text { Usability der Plattform und } \\
\text { Socia-bility (Etiquette, etc.) }\end{array}$ & $\begin{array}{l}\text { Transparentes Management } \\
\text { der Teilnehmer/Etablierung } \\
\text { v. Subgemeinschaften }\end{array}$ \\
\hline Erprobbarkeit & Beteiligung potenzieller & $\begin{array}{l}\text { Beteiligung der potenziellen } \\
\text { Anwender an der Entwick- } \\
\text { lung }\end{array}$ & & Evaluierung der Gemeinschaft \\
\hline Beobachtbarkeit & Anwender an der Analyse & $\begin{array}{l}\text { Sicherstellung der Trans- } \\
\text { parenz der Designentschei- } \\
\text { dungen }\end{array}$ & \multicolumn{2}{|c|}{$\begin{array}{l}\text { Gewährung von Anreizen, } \\
\text { Weiterentwicklung der Gemeinschaft, } \\
\text { Qualitätssicherung }\end{array}$} \\
\hline
\end{tabular}


Implementierung und Betrieb: Auf wichtige Aspekte der Einführung von Communityplattformen weist der Ansatz der "technology-use mediation" hin. Dieser Ansatz betont, dass auch eine ausgereifte technische Unterstützung nicht effektiv genutzt wird, wenn sie nicht in die Interaktionspraktiken ihrer Umgebung eingebettet wird [OYOF95]. Um die zentrale Anlaufstelle für die Interaktion ihrer Mitglieder zu bilden, müssen Funktionalitäten und Regelungen eingeführt, kommuniziert und aufrechterhalten werden. Erfolgsfaktoren und Handlungsanweisungen bezüglich des Betriebs von VCs betreffen vor allem die Bereiche Communityentwicklung inklusive der Umsetzung von vertrauensunterstützenden Komponenten, sowie Teilnehmer- und Anreizmanagement und die Qualitätssicherung der VC.

Controlling und Evolution: Eine erfolgreiche Implementierung bedeutet, dass für den einzelnen Teilnehmer eine intensive Nutzung der VC gemäß eigener Ziele ermöglicht wird. Um die Vielfalt und das Wachsen der VC zu fördern, sind SubCommunitys zu etablieren und zu pflegen. Eine Daueraufgabe stellt auch in dieser Phase die Qualitätssicherung des Angebots dar. Darüber hinaus sind VCs als viel versprechendes Geschäftsmodell bezeichnet worden [BuHa00, 237; HaAr97]. Je nach Art der zu etablierenden Community stellen die Verwirklichung des Geschäftsmodells sowie die systematische Evaluation und Evolution der Community Erfolgsfaktoren für diese Phase ihres Lebenszyklus dar.

Diese Erfolgsfaktoren und Gestaltungsmöglichkeiten werden im CB\&CM-Vorgehensmodell zusammenfassend dargestellt (vgl. Bild 1).

\section{Methodisches Vorgehen - die Pilotierung einer sozio- technischen Innovation}

Da der Untersuchungsgegenstand VCs für Patienten kaum im deutschen Gesundheitswesen anzutreffen ist und damit auch das Vorverständnis des Forschungsgegenstandes „VCs für Patienten in Deutschland“ noch zu gering ist, als dass sinnvolle Hypothesen oder ein sinnvolles Model aufgestellt werden könnten, blenden viele Disziplinen ein derartiges Untersuchungsfeld aus. Die Wirtschaftsinformatik hingegen bietet dem Forscher mit der Methode der „Pilotierung IT-basierter Innova-

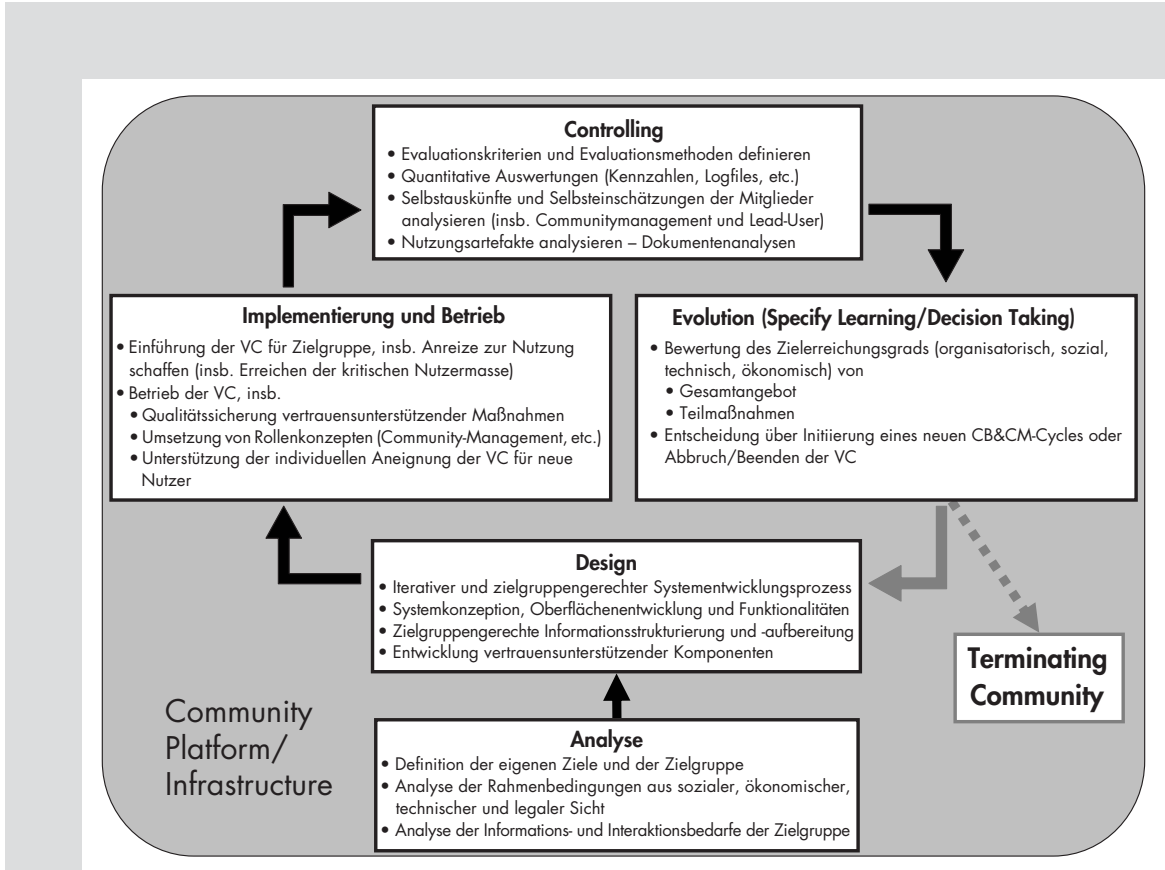

Bild 1 Community-Building- und Community-Management Vorgehensmodell

tionen“ die Möglichkeit, selbst die zu untersuchende sozio-technische Innovation zu schaffen und anschließend zu evaluieren.

\subsection{Kernpunkte der Methode}

Pilotprojekte entwickeln und implementieren technologische Innovationen in ihrem natürlichen organisationalen und sozialen Umfeld [ScKro0, 133]. Da es sich bei der Pilotierung um eine Spielart der Aktionsforschung handelt [FKKT98], wird im Folgenden nur auf die wichtigsten Unterschiede eingegangen.

Pilotprojekte starten mit einem realweltlichen Problem und durchlaufen idealtypisch einen Zyklus. Im Unterschied zu einem in sozialwissenschaftlicher Tradition stehenden Aktionsforschungsprojekt wird bei einer Informationsystem-Pilotierung bspw. die Intervention innerhalb eines Forschungsprozesses nicht aus rein sozialer Perspektive, sondern aus sozio-technischer, oftmals sogar aus rein technischer Perspektive betrachtet. In einem typischen Pilotierungsprojekt wird eine neue, innovative Software entwickelt und im Feld eingeführt. Die Anwendung/Nutzung der Software führt dann einerseits zu einer Verbesserung der Software und andererseits zu einem Eingriff in ein soziales System mit Veränderungen/Verbesserungen der realen Welt. Auf diese Art wird der traditionelle
Prototypingansatz der Informationssystementwicklung mit der Intervention in ein reales soziales System verbunden.

\subsection{Potenziale und Schwierig- keiten in der Anwendung}

Bei der Pilotierung eines Informationssystems handelt es sich um eine geplante Innovationsgestaltung, deren Realisierung der Erarbeitung von Regeln zur Gestaltung und Handhabung noch nicht realisierter Systeme entspricht (zur Forderung nach Pilotierungsforschung in der Betriebswirtschaftslehre siehe bspw. [Szyp71], zur Beschreibung von Feldexperimenten/Pilotprojekten als Innovationstest siehe bspw. [Witt97]). Die Gestaltung dieser Systeme oder anders ausgedrückt dieser sozio-technischen Innovationen ist nicht nur eine dedizierte Aufgabe der Wirtschaftsinformatik [HeKH01; KöHP95], sondern auch ein wertvoller Beitrag für die Disziplin.

Pilotprojekte haben zwei entscheidende Vorteile [Schw00, 189f.]: Zum einen dienen sie als Innovationsprüfstein, da Machbarkeit und Wirkungen nur durch Erprobung abgeschätzt werden können, andererseits können sie als Innovationsbeispiel andere potenzielle Interessenten unter Umständen zur Nachahmung animieren (sog. „Leuchtturmprojekte").

Es gelten aber bei Pilotprojekten die gleichen Einschränkungen und Hindernis- 
se wie bei Feldexperimenten allgemein: Die fehlende Möglichkeit der Kontrolle von Störfaktoren und die unklaren Wirkungszusammenhänge im natürlichen Feld verringern die interne Validität, im Gegenzug wird meist eine höhere externe Validität postuliert ScHE99, 216f.].

Weiterhin ist oftmals die Identifikation von Ursache-Wirkungszusammenhängen in der Tiefe nicht möglich. Das tiefere Verständnis eines Forschungsproblems, das mit einem Pilotprojekt erreicht werden kann, rührt dementsprechend nicht aus dem Verständnis einiger weniger Variablen in der Tiefe her, sondern aus dem reichen Bild des Projektergebnisses insgesamt [ScKr00, 138f.]. Darüber hinaus erfordert die Durchführung einer wissenschaftlichen Pilotierung viel Erfahrung, da die Anforderungen an einen Aktionsforscher generell als hoch einzuschätzen sind. Die Tatsache, dass viele der in der Wirtschaftsinformatikforschung entwickelten Prototypen nicht im Feld erprobt werden, liegt eher an dem damit verbundenen Aufwand, den hohen Anforderungen an die Forscher und dem Bedarf nach einem geeigneten organisationalen bzw. institutionellen Umfeld, in dem das Pilotprojekt stattfinden kann, als an einer fehlenden wissenschaftlichen Fundierung der Pilotierungsforschung [Schw00, 187].

Eines der größten Potenziale einer Pilotierung ist die Entwicklung eines reichen, holistischen Verständnisses der untersuchten Innovation und des Wechselspiels zwischen Nutzung und Aneignung derselben in ihrem natürlichen Umfeld. Weiterhin erlaubt die Pilotierung die Erarbeitung von vielfältigen und sehr ergiebigen empirischen Daten, welche die Qualität der Evaluation der Innovation maßgeblich verbessern können und Erkenntnisse erlauben, die durch andere Forschungsmethoden überhaupt nicht möglich gewesen wären. Im Falle einer VC sind hier bspw. der Zugang zu jedweden Nutzungsdaten wie Logfiles (natürlich nur nach vorheriger Einverständniserklärung der Nutzer) oder die Möglichkeit extensiver Beobachtungen, Befragungen oder der Durchführung von Experimenten mit Gestaltungsparametern (Farben, Funktionalitäten, etc.) zu nennen. Darüber hinaus ist das Entwickeln von Gestaltungsempfehlungen basierend auf einem gesicherten Erkenntniszusammenhang durch den iterativen Lernprozesses bei der Pilotierung möglich.

\section{Fallbeispiel: Pilotierung Virtueller Communitys für Krebspatienten}

Die hier beschriebene Pilotierung wurde entlang des dargestellten CB\&CM-Vorgehens durchgeführt und es wird folgend schwerpunktmäßig auf die Besonderheiten der jeweiligen Phasen eingegangen.

\subsection{Rahmenbedingungen Virtueller Communitys für Krebs- patienten aus Deutschland}

Krebserkrankungen sind mit ca. 395.000 Neuerkrankungen jährlich die zweithäufigste Todesursache in Deutschland [Deut06]. Umso erstaunlicher ist, dass bis 2001 kaum Informations- und Interaktionsangebote für Patienten im deutschsprachigen Internet $\mathrm{zu}$ finden waren [DKLK01].

\subsubsection{Bedarfe von Krebspatienten}

Die Diagnose der Krebserkrankung trifft den Patienten meist völlig unerwartet. Die oft sich anschließenden langwierigen Behandlungszyklen dominieren den Tagesablauf und die mittelfristige Lebensplanung. Arbeit, Privatleben und Familie verlieren aufgrund dieser veränderten Lebenssituation an Priorität. Ausgelöst durch die lebensbedrohende Diagnose stürzt der Patient oftmals auch psychisch in eine Krise. Diese Krisen lösen einen starken Bedarf nach Verarbeitung und Bewältigung der neuen Lebenssituation aus [Mada97]. Verstehen, was mit dem Betroffenen passiert, setzt Information über die Krankheit voraus. Doch der Wunsch nach harten Fakten ist nur ein Teil dieser Prozesse. Neben dem Bedarf an Informationen kann es auch ein Verlangen nach Verständnis und emotionaler Unterstützung durch andere Betroffene sowie Interaktion mit diesen geben [Mada97].

Krebspatienten entwickeln oft einen hohen Informationsbedarf, z. B. nach der Diagnose oder während einer Therapie [BiDe96; MiSu99]. Dieser Bedarf kann aus einer asymmetrischen Informationsverteilung zwischen Arzt und Patient resultieren.

Welche Informationsarten von den $\mathrm{Pa}$ tienten im Detail nachgefragt werden, zeigen neuere Forschungsergebnisse. Kaminski et al. [KTCM01] identifizieren unter anderem eine starkes Interesse an Informationen aus unterschiedlichen Bereichen, z. B. Auswirkungen der Erkrankung auf andere Lebensbereiche, wie Arbeit, Familie oder Sexualität (ähnliche Ergebnisse auch bei [BiDe96; ShKn03]). Mit diesen Fragen ist aber der behandelnde Arzt oft überfordert. Dabei geht es den Patienten nicht nur um medizinische Kompetenz im engeren Sinne, sondern vor allem um Kommunikation und einen menschlichen Umgang mit dem individuellen Leiden der Einzelnen [Hill01]. Wechselseitige Akzeptanz, mehr Zuwendung, Empathie und Ganzheitlichkeit sowie eine höhere Qualität und bessere Zusammenarbeit zwischen den verschiedenen Akteuren im Behandlungsprozess werden als Problemfelder genannt.

Oftmals ist auch im direkten Umgang mit betroffenen Menschen das Bedürfnis nach Empathie und zwischenmenschlicher Interaktion spürbar. Die Interaktion mit Gleichgesinnten/Menschen mit gleichem Schicksal kann eine große Rolle spielen [Forb01]. Die Bedarfe der Patienten beschränken sich nicht nur auf reine Fakten, vielmehr wollen sie, dass Informationen begründet und hinsichtlich ihres Krankheitsbildes ausgewertet und für ihre Lage interpretiert werden [vgl. hierzu auch MiSu99; Moel96]. Um die neue Situation $\mathrm{zu}$ verarbeiten, das Wissen um Behandlungsmöglichkeiten oder Informationen über Heilungschancen $\mathrm{zu}$ diskutieren, braucht der Betroffene Gesprächspartner. Besonders interessiert ist er an Erfahrungen oder ähnlichen Fällen [BiDe96]. Zwar ist im Falle einer Krebserkrankung nicht immer mit einem aktiven Umgang in Form von Informationssuche und Interaktion mit anderen Menschen zu rechnen. Dass aber Menschen, die aktive Krankheitsbewältigung mittels Informationssuche und Austausch betreiben, einen Vorteil hieraus ziehen können, ist in der Literatur mehrfach belegt [vgl. hierzu bspw. $\mathrm{McHH01]}$.

\subsubsection{Gesetzliche Rahmen- bedingungen bei medizinischen Internetangeboten in Deutschland}

Das Gesundheitswesen ist in Deutschland ein gesetzlich hoch reglementierter Bereich. Beteiligte am Gesundheitswesen unterliegen aufgrund ihrer Berufszugehörigkeit oder Anstellung bei einer bestimmten Einrichtung unterschiedlichen Rechtsnormen. Medizinische und Patienten-Informationsangebote im Internet unterliegen den allgemeinen Anforderungen an Internetangebote. Es bestehen nur wenige Sonderregelungen, die beim Angebot medizi- 
nischer Inhalte zu beachten sind [DiNG03, 95]. Es sind insbesondere der Mediendienste-Staatsvertrag, das Teledienstegesetz (TDG), sowie die Novellierungen im Rahmen des Gesetzes über die rechtlichen Rabmenbedingungen für den elektronischen Geschäftsverkehr (BGBl. I, S. 3721) zu berücksichtigen.

Besondere Beachtung verdienen insb. die folgenden Aspekte: Die Regelung der Haftung des Inhaltes fremder Websites (Verlinkung), die Berücksichtigung heilberufsrechtlicher Grenzen (in Form der Unterscheidung zwischen Information und Beratung), Aspekte des Datenschutzes in Zusammenhang mit Telediensten sowie Besonderheiten der Weitergabe von Fachinformationen an nicht autorisierte Laien. Diese rechtlichen Rahmenbedingungen haben dergestalt Einfluss auf Internetangebote in Deutschland, dass sie bspw. entsprechende Nutzer- und Nutzungsvereinbarungen sowie Disclaimer erfordern. Außerdem führen sie dazu, dass individuelle Beratungsangebote für $\mathrm{Pa}$ tienten über das Internet nicht zulässig sind und nur durch Informationsangebote annähernd ersetzt werden können [vgl. KRSB05].

\subsection{Zentrale Designelemente bedarfsgerechter Virtueller Communitys für Patienten am Beispiel von krebsgemeinschaft.de}

Wichtige Designelemente einer VC für $\mathrm{Pa}$ tienten sind die Informationsaufbereitung, die Funktionalitäten, Benutzerfreundlichkeit (Usability) und Accessibility (i. S. v. Barrierefreiheit) des Angebots sowie der Einsatz vertrauensunterstützender Komponenten [Leim05].

\subsubsection{Informationsdienste}

Der Schwerpunkt im Bereich Informationsdienste liegt in der großen Menge an redaktionell aufbereitetem und qualitätsgesichertem Inhalt zum Thema Brustkrebs. Die Erarbeitung einer Struktur für diesen komplexen Inhalt ist eine der zentralen Herausforderungen neben der laienverständlichen Aufbereitung.

Im Rahmen einer iterativen Entwicklung und des Durchlaufens mehrerer Runden von Expertengesprächen wurde die folgende Kategorisierung erarbeitet und in folgende Rangfolge der erwarteten Relevanz für die Nutzer gebracht: a) Krebs behandeln (Therapie, Nachsorge); b) Krebs erkennen (Früherkennung, Diagnosemetho- den); c) Krebs erforschen (Studien, Zahlen und Daten); d) Leben mit Krebs (Sport und Ernährung, Familie und Sexualität) und e) Erfabrungen mit Krebs (Betroffene berichten). Die Aufteilung der Oberkategorien in kleinere Untereinheiten soll dem Nutzer helfen, die dargebotenen Informationen einfach strukturiert schneller wahrzunehmen und besser kognitiv zu verarbeiten.

\subsubsection{Funktionalitäten}

Im Bereich der Funktionalitäten werden die Dienste Diskussionsforum, Fragen an Experten, Kontaktsuche und Chat angeboten. Darüber hinaus werden über die personalisierte Startseite der Mitglieder „persönliche“ Interaktionsdienste wie ein internes Mail-System oder das eigene Gästebuch, oder aber Informationsdienste wie Freundeslisten und Awareness-Funktionen wie „Freunde online“, „Nutzer im Chat" oder „Mitglieder in der krebsgemeinschaft.de" bereitgestellt (vgl. Bild 2). Diese Funktionalitäten wurden im Rahmen eines iterativen Entwicklungsmodells mit mehreren Fokusgruppen als für die Zielgruppe relevant identifiziert [ArLK03].

Das Diskussionsforum ermöglicht den asynchronen Austausch der Mitglieder untereinander. Damit unterstützt es neben der Kommunikation auch den Prozess des Kennenlernens der Mitglieder untereinander und fördert somit den Aufbau und die Pflege einer Gemeinschaft.

Der Dienst "Fragen an Experten“ ist ein Diskussionsforum, in dem die Nutzer Fragen stellen und nur die Experten antworten können. Darüber hinaus sind die Dauer einer Fragerunde und das Thema vorgegeben. Auf diese Weise wird der Aufwand für die beantwortenden Experten reduziert und besser planbar. Die Kontaktsuche hat zum Ziel, den Mitgliedern von krebsgemeinschaft.de eine Unterstützung bei der Suche nach Menschen in ähnlichen Lebenslagen und/oder mit ähnlichen Interessen zu bieten. Diese einfache Möglichkeit der Kontaktaufnahme mit anderen Mitgliedern soll den persönlichen Erfahrungs- und Meinungsaustausch sowie die Bildung eines Gemeinschaftsgefühls fördern.

Der Chat ist eine synchrone Echtzeitkommunikation und bietet den Nutzern die Möglichkeit, miteinander zeitgleich in Kontakt zu treten. Um in der virtuellen Community die Wahrscheinlichkeit, einander zu treffen zu erhöhen, wurden im betrachteten Fall feste Zeiten für den Chat vorgegeben [BrMW00, 137].

\subsubsection{Benutzerfreundlichkeit (Usability)}

Neben der Bedarfsgerechtigkeit der Inhalte kommt der Benutzerfreundlichkeit besondere Bedeutung zu. Es spielen Aspekte wie Layout, Farb-, Grafik- und Schriftgestaltung sowie Ergonomie eine wichtige Rolle. Diese Aspekte der Usability sind immer kontext- und verwendungsspezifisch, daher kann es keine detaillierten allgemeingültigen Regeln hierfür geben. Usability und Gesamteindruck haben außerdem entscheidenden Einfluss darauf, ob Websites Glaubwürdigkeit und Vertrauen ausstrahlen [FSDM02]. Aus der Vielzahl unterschiedlicher Usability-Prinzipien lassen sich folgende Metaprinzipien herausfiltern:

Aufgabenangemessenbeit: Ein Dialog mit einem System gilt dann als angemessen, wenn er die Erledigung der Arbeitsaufgabe des Benutzers unterstützt, ohne ihn durch Eigenschaften des Dialogsystems unnötig zu belasten.

Erwartungskonformität: Ein System verhält sich dann erwartungskonform, wenn jedes Element/jeder Teil dem Benutzer das Design und den Inhalt präsentiert, den er an dieser Stelle erwartet.

Konsistenz: Ein einheitliches Erscheinungsbild eines Systems in einem Kontext wird als Konsistenz bezeichnet. Konsistenz führt zu Usability, weil der Benutzer auf bereits Gelerntes zurückgreifen kann und sich nicht neu anpassen bzw. neu lernen muss. Es ist eines der wichtigsten Usability-Prinzipien [Mayh92, Spol01, Niel02]

Visibility: Gute Mensch-MaschineSchnittstellen haben gut sichtbare Bedienungselemente. Jedes Bedienelement (Knopf, Schaltfläche, etc.) kontrolliert idealerweise nur eine Funktion.

\subsubsection{Barrierefreiheit (Accessibility)}

Wenn das Internet eine Weitergabe von Information als Text sicherstellt, kann es bei physischen Behinderungen auch dazu beitragen, diese $\mathrm{zu}$ überwinden, indem die Behinderung eines Sinnesorgans durch die Nutzung eines anderen ersetzt wird. So können bspw. die Inhalte einer Seite durch entsprechende Hilfsmittel für blinde oder sonst körperlich eingeschränkte Menschen nutzbar gemacht werden [WEBf06]. Dies ist für die Zielgruppe der Krebspatienten besonders wichtig, da sie je nach Behandlungsstadium mit zeitweise eingeschränkter Mikromobilität und körperlichen Behinderungen umzugehen hat. Das Behindertengleichstellungsgesetz (BGG) richtet sich gegen die Benachteiligung von behinderten 


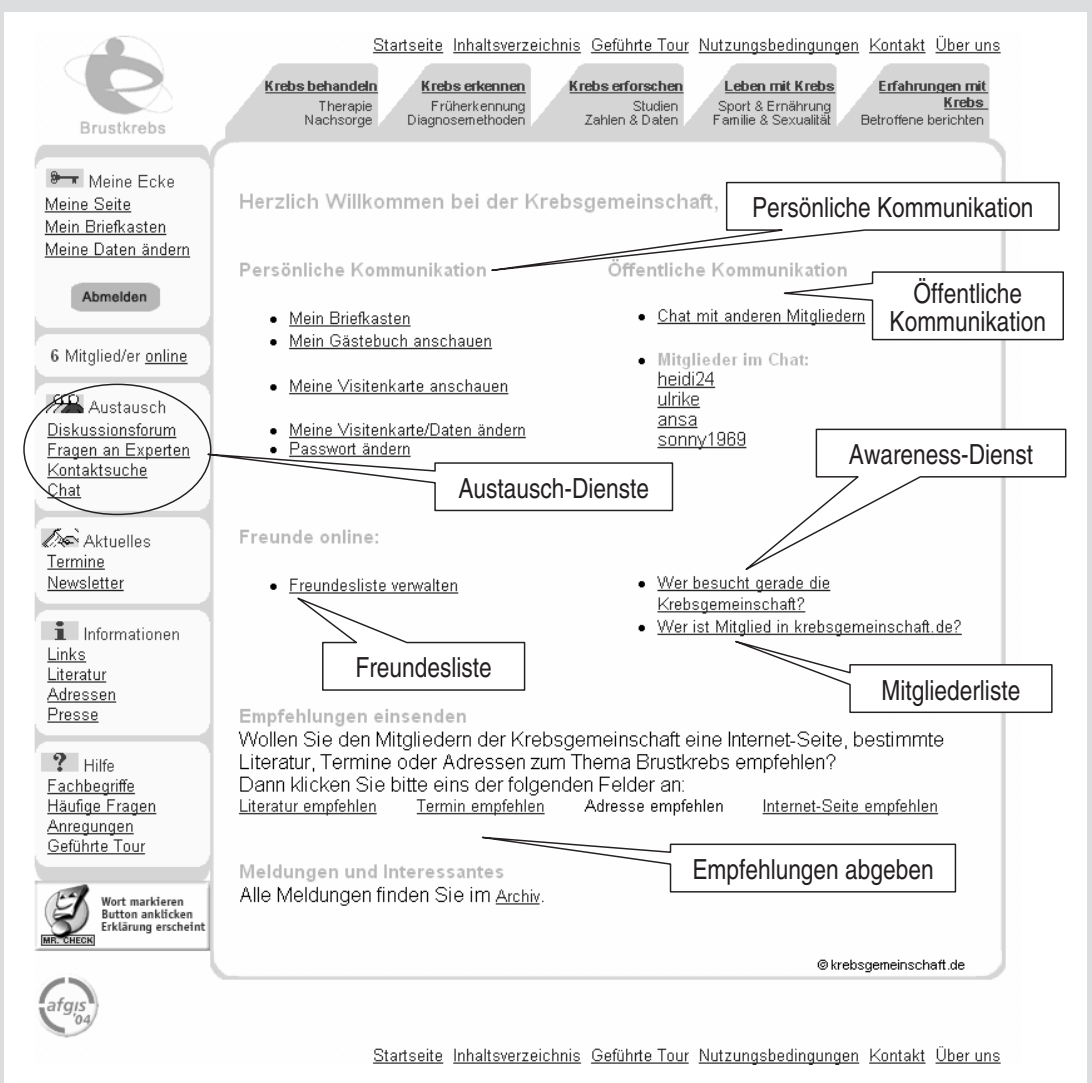

Bild 2 Informations- und Interaktionsdienste sowie grafische Aufbereitung der persönlichen Startseite der Mitglieder von www.krebsgemeinschaft.de (Quelle: [Leim05, 201])

Menschen. $₫ 11$ BGG schreibt vor, dass Träger öffentlicher Gewalt ihre Internetauftritte und -angebote sowie die von ihnen zur Verfügung gestellten grafischen Programmoberflächen, die mit Mitteln der Informationstechnik dargestellt werden, so gestalten müssen, dass sie von behinderten Menschen grundsätzlich uneingeschränkt genutzt werden können. Eine barrierefreie Internetseite ist bei Beachtung der entsprechenden Gestaltungsempfehlungen für barrierefreie Internetseiten [vgl. Leim05, $183 \mathrm{ff}$.] mit den derzeit zur Verfügung stehenden Hilfsmitteln problemlos lesbar und navigierbar.

\subsubsection{Vertrauensunterstützende Komponenten}

Neben einer benutzerfreundlichen Oberfläche und Nutzen stiftenden Diensten der Communityplattform selbst ist die Vertrauenswürdigkeit des Angebotes ein zentraler Erfolgsfaktor. Entscheidende

\subsection{Akzeptanz und Nutzen von krebsgemeinschaft.de}

\subsubsection{Evaluationskriterien und Evaluationsmethoden}

Um Aussagen über die Wirksamkeit der im Rahmen der Pilotierung von krebsgemeinschaft.de durchgeführten Maßnahmen machen zu können, sind Bezugsgrößen für den Erfolg sowohl der einzelnen Maßnahmen als auch der Gesamtheit aller Maßnahmen (also der gesamten Virtuellen Community) zu bestimmen. Eine Erfolgsmessung bzw. eine Ursache-Wirkungskette ist bei vielen Einzelmaßnahmen jedoch nicht möglich, da sie nicht losgelöst von anderen Einflussgrößen auf den Nutzer wirken. Oftmals ist sogar nur die Summe mehrerer Maßnahmen und Einflussgrößen als Gesamtheit erfassbar und bewertbar. Bild 3 verdeutlicht die wichtigsten zuvor dargestellten Designmaßnahmen und ihre Wirkung als Gesamtheit auf den Erfolg bzw. die Akzeptanz und den Nutzen von krebsgemeinschaft.de

Spezifischere Indikatoren für den Erfolg Virtueller Communitys lassen sich auf unterschiedlichen Ebenen finden, wenngleich es an systematischen und operationalisierbaren Vorschlägen mangelt. Für die Evaluation bieten sich sowohl Techniken aus der „klassischen“ Sozialforschung als auch aus der Onlineforschung an. Im Rahmen dieser Untersuchung wurden LogfileAnalysen, Onlinebefragungen, Dokumentenanalysen und Beobachtungen verwandt.

Um nicht nur eine Momentaufnahme der Einschätzungen der Mitglieder der VC abzubilden wurde der identische Befragungssapparat nach 18 Monaten erneut eingesetzt. Diese Longitudinalergebnisse sollen über einen längeren Zeitraum stabile Aussagen ermöglichen und die Nachhaltigkeit des Community-Engineering-Ansatzes überprüfen.

\subsubsection{Empirische Ergebnisse}

Zur Evaluation der VC krebsgemeinschaft.de wurden unterschiedliche Methoden verwendet. Alle Angaben beziehen sich (sofern keine anderen Angaben gemacht werden) auf die Zeitspanne vom 19. 8. 2002 (Onlineschaltung der VC) bis zum 30. 8. 2005.

Den Kern einer VC bildet die Interaktion zwischen den Mitgliedern, die entweder in Chats oder Diskussionsforen stattfindet. Dabei muss beachtet werden, dass die Logfile-Analyse und insgesamt rein quantitativen Werte zur Beurteilung 
einer VC nicht hinreichend sind. So kann bspw. ein kleiner Kreis von aktiven Nutzern (wie dieser sich auch bei krebsgemeinschaft.de herausgebildet hat) viel stärker zum „Leben“ einer Community beitragen als ein großer Kreis passiver Nutzer, sog. „Lurker" [Pree00]. Aus diesem Grund werden neben Logfile-Analysen auch qualitative Analysen von krebsgemeinschaft.de durchgeführt, u. a. in Form von Onlinebefragungen unter den Nutzern, Beobachtungen, Inhaltsanalysen und Auswertungen des Interaktionsverhaltens in der VC. Die jeweiligen Teilergebnisse werden in einer Methodentriangulation miteinander verglichen.

Hintergrundinformationen $\mathrm{zu}$ den Nutzern von krebsgemeinschaft.de (Stand Aug. 2005): Das Durchschnittsalter der Nutzer beträgt 43,7 Jahre, es sind $91 \%$ Frauen, $73 \%$ sind selbst Krebspatienten, $18 \%$ Angehörige. Bei der Registrierung erklärten $67 \%$, geringe bis gar keine Interneterfahrung zu besitzen. $41 \%$ gaben an, über Empfehlungen der am Pilotierungsprojekt beteiligten Institutionen auf das Angebot aufmerksam geworden zu sein.

Quantitative Nutzungszahlen: Zur Bewertung der „Member-to-member interaction" werden in Anlehnung an [Coth00, 18] die Kriterien Visits, Seitenaufrufe, aktive Nutzer (schreibend), passive Nutzer (nur lesend) und Anzahl der Postings herangezogen. Sowohl die Anzahl an Visits als auch an Seitenaufrufen steigt kontinuierlich, parallel dazu verhält sich die Anzahl an aktiven, d. h. Postings im Forum erstellenden Nutzern als auch die Anzahl an Lurkern, den nur lesenden Besuchern der Community. Auffällig ist, dass das Wachstum der aktiven Nutzer halb so stark ausfällt wie das Wachstum der Lurker.

Gesamtbeurteilung: Insgesamt beurteilten die Teilnehmer zweier Nutzerumfragen den Aufbau von krebsgemeinschaft.de positiv. Insbesondere aus der Longitudinalperspektive wird deutlich, dass sich die anfänglich positive Einschätzung über die Zeit hinweg verbessert und verstetigt hat. Dies überrascht nicht, da es sich bei den Teilnehmern überwiegend um Personen handelt, die das Angebot stark nutzen. Eine freiwillige wiederholte Nutzung kann für sich genommen schon als positive Beurteilung des Angebots gewertet werden. Deutlich fällt auf, dass die ursprünglich (Befragung 1, 10/2003) als unzureichend eingeschätzte technische Performanz des Systems vor Befragung 2 (1/2005) verbessert wurde.

Folgende Antwort auf die Frage „Bitte beschreiben Sie kurz, wie sich die Mitglied-

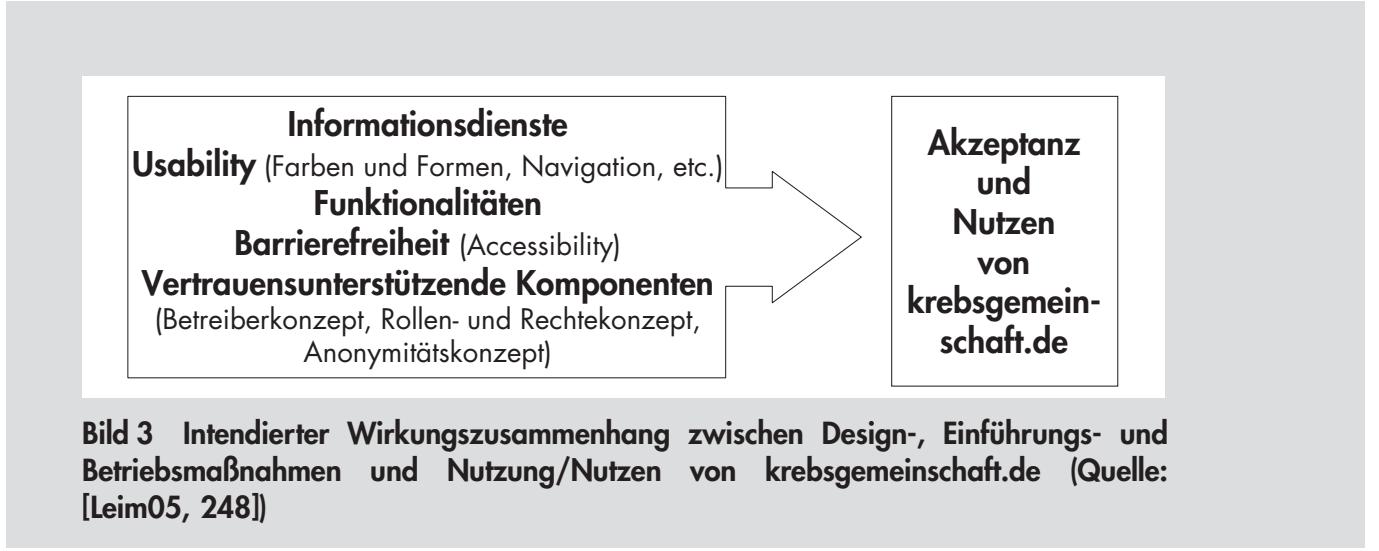

schaft bei krebsgemeinschaft.de auf ihre Situation auswirkt/ausgewirkt hat." verdeutlicht die Bedeutung des Angebots für die Mitglieder:

„Der Austausch mit Betroffenen ist sebr wobltuend. Gerade nach der Krankbeit geben alle davon aus (Familie, Beruf), dass das Leben wieder so ist wie vorher. Dem ist nicht so!!! Natürlich will man nicht ständig über das (schlechte) Befinden sprechen und irgendwie will das ja auf Daner auch niemand hören. Deshalb ist der Austausch mit Betroffenen wichtig, es wird Mut gemacht und es hilft zu wissen, dass man in dieser Situation nicht alleine auf der Welt ist. Es ist auch sebr beeindruckend zu lesen, wie manche Franen mit ihrem Schicksal umgehen. Das weckt den Kämpfergeist und hilft, den Heilungsprozess oder auch Rückschläge besser zu verarbeiten."

Vertrauen: Bei der Konzeption und beim Aufbau von krebsgemeinschaft.de wurden vertrauensfördernde Komponenten konzipiert und in die VC integriert. Der Prozess der Vertrauensbildung sollte durch diese Komponenten unterstützt werden, um so zum erfolgreichen Aufbau und Betrieb der Gemeinschaft beizutragen. Die Ergebnisse einer Onlineumfrage, die zum Ziel hatte, das Vertrauen in die VC krebsgemeinschaft.de zu messen [LeEK05], bestätigt den Erfolg dieser vertrauensbildenden Komponenten. Sowohl das Vertrauen in die Betreiber von krebsgemeinschaft.de, hier vor allem in deren fachliche Kompetenz, als auch das Vertrauen in die anderen Mitglieder der VC, hier vor allem in das wahrgenommene Wohlwollen, sind hoch. Auch die Handlungsweisen der befragten Mitglieder entsprechen diesem Ergebnis: Sie sind sowohl bereit, Informationen, die innerhalb der Community zur Verfügung stehen, zu nutzen, als auch selbst persönliches Erfahrungen und eigenes Wissen zur Verfügung zu stellen. Damit zeigen sie, dass sie sowohl darauf vertrauen, dass persönliche Informationen nicht missbraucht werden, als auch darauf, dass bereits in der Gemeinschaft vorhandenen Informationen und Daten als richtig erachtet werden. Darüber hinaus konnte nachgewiesen werden, dass die Befragten zum Großteil sogar entsprechend dieser Informationen handeln (vgl. [LeEK05]).

Ausbildung sozialer Netzwerke: In der VC haben sich von Anfang an und über die Zeit hinweg stabile und reiche soziale Strukturen ausgebildet. Die Analyse der Gästebücher der 20 nach Postings aktivsten Community-Mitglieder zeigt ein feinmaschiges soziales Netzwerk mit einer Vielzahl von Mitgliedern. Selbst wenn einzelne Knoten aus diesem Netzwerk verschwinden sollten bliebe das soziale Gebilde intakt. Dies deckt sich auch mit den Erfahrungen bei Todesfällen von aktiven Mitgliedern der Gemeinschaft. Die Stärke und vor allem die Stabilität der ausgebildeten sozialen Beziehungen in der Community über längere Zeit hinweg werden von diesen Nutzern als stark und für ihre Krankheitsbewältigung als sehr hilfreich eingeschätzt [ScLK06]. Insbesondere die Knoten mit vielen Kanten sind von Bedeutung, da dies Mitglieder sind, die aufgrund Ihrer Vielzahl an Beziehungen in der VC eine starke Integrationsleistung erbringen, entsprechend können sie vom Communitymanagement gefördert werden und/oder in die Aufbau- und Ablauforganisation involviert werden.

\subsubsection{Zusammenfassung der Evaluationsergebnisse}

Die Evaluation von krebsgemeinschaft.de über mehr als zwei Jahre mittels unterschiedlicher Methoden und Messgrößen zeigt, dass die Virtuelle Gemeinschaft ein erfolgreiches Angebot ist und für die $\mathrm{Pa}$ - 


\section{Gesamtbeurteilung auf Basis von Nutzerbefragungen ( 1 =stimme voll und ganz $\mathrm{zu}, 5=$ stimme überhaupt nicht $\mathrm{zu}$ )}

Die Seiten werden schnell angezeigt.

Es ist leicht ersichtlich, von wem welche Informationen stammen (z. B. Team der Krebsgemeinschaft, andere...).

Es dauert nicht lange, gewünschte Inhalte zu finden.

Es fiel mir von Anfang an leicht, die Seiten zu nutzen.

Die Erklärungen (Hilfe) helfen mir, mich zurecht zu finden.

Die Farben der Seiten sind angenehm.

Die Schrift ist immer gut lesbar.

Die Seiten sind übersichtlich.

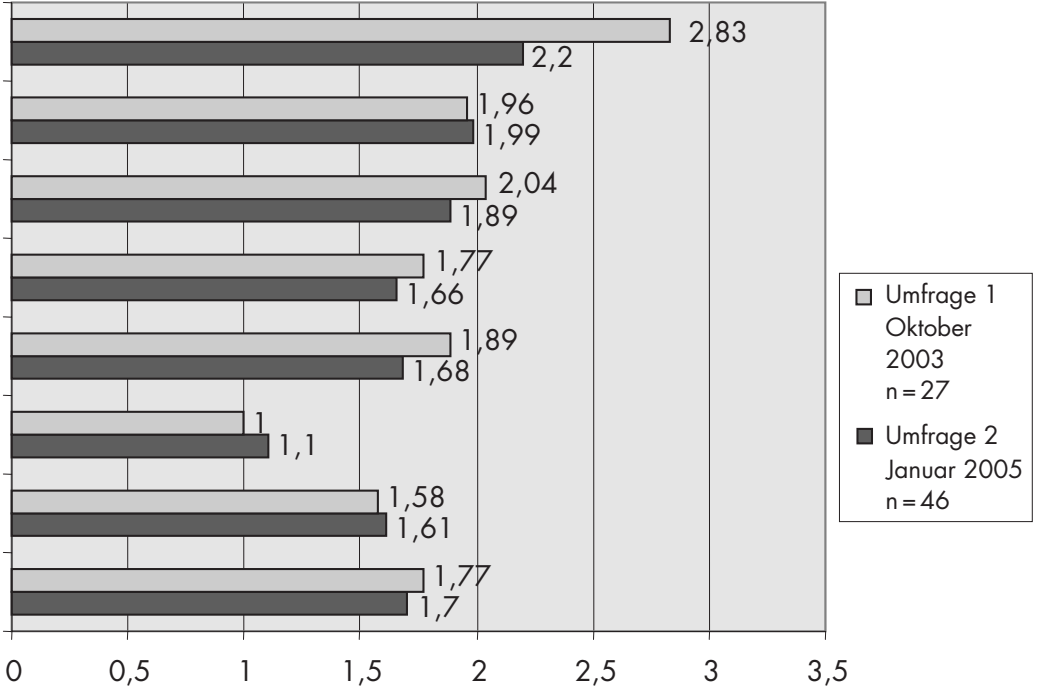

Bild 4 Ergebnisse von Nutzerbefragungen in 2003 und 2005 zur Gesamtbeurteilung von krebsgemeinschaft.de

tienten einen hohen Nutzen hat. Mit dieser Pilotierung liegt ein empirisch longitudinal nachweislich erfolgreicher Vorschlag für das Community-Engineering im deutschen Gesundheitswesen vor.

Der Erfolg des Angebotes lässt sich anhand verschiedener quantitativer und qualitativer Indikatoren verdeutlichen. Die Mitgliederentwicklung verzeichnet über den Betrachtungszeitraum einen deutlichen

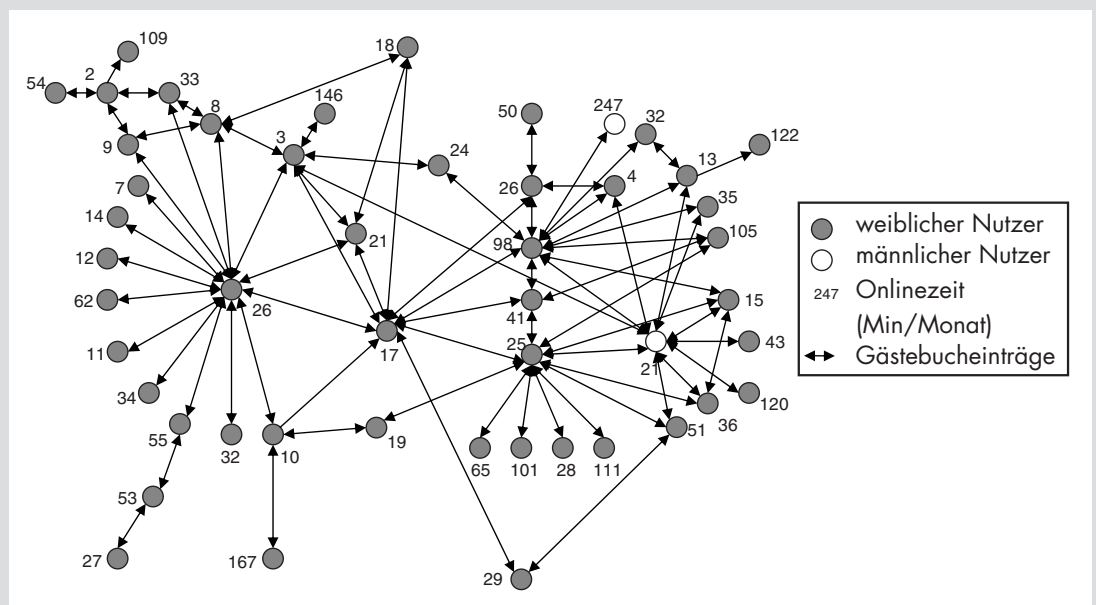

Bild 5 Visualisierung sozialer Kontakte in krebsgemeinschaft.de anhand der Gästebucheinträge im Juni 2005 und konstanten Anstieg. In der VC kann zwischen einer festen „Kerngemeinschaft" aus kontinuierlich aktiven Teilnehmern und einer erweiterten „äußeren Schale“ mit passiven Mitgliedern unterschieden werden. Grundlegende Verhaltensmuster und Normen, die sich in der VC herauskristallisiert haben, sind insbesondere das Prinzip der Wechselseitigkeit und des aktiven Austausches von Informationen, Empathie und

Unterstützung. Das für diese Verhaltensweisen notwendige zugrunde liegende Vertrauen ist sowohl gegenüber anderen Mitgliedern, als auch gegenüber den Betreibern der VC andauernd empirisch belegbar relativ hoch ausgeprägt. Konzept und Einführung vertrauensunterstützender Komponenten haben sich demnach als erfolgreich erwiesen. Umgekehrt zeigt sich im Hinblick auf negative Ereignisse, dass die Anzahl an Verstößen gegen die Regeln der Gemeinschaft oder Vorfällen, die als grob oder unhöflich einzustufen sind, ausgesprochen gering ist. Ein Indiz für relativ hohe Treue und Bindung der Mitglieder an die VC ist, dass der initiale Kern der Mitglieder seit Gründung der VC immer noch aktiv ist. Die empfundene Stärke der virtuellen sozialen Beziehungen der Mitglieder untereinander unterstreicht dies deutlich.

Bezogen auf die Bedarfsgerechtigkeit der Communityplattform sind die Mitglieder größtenteils zufrieden, obgleich einzelne Funktionalitäten von den Mitgliedern als unterschiedlich nutzenstiftend bewertet werden. Beispielsweise wird das Design bei Oberflächenentwicklung und Funktionalitäten auch nach langer Nutzung als bedarfsgerecht und gut beurteilt. Allerdings verdeutlichen die Ergebnisse der Befragungen auch, dass einzelne Eigenschaften der VC vielen Nutzern auch nach langer Existenz nicht bekannt sind und daher nicht 
genutzt werden (z. B. die Funktionalität der Kontaktsuche). Vergleicht man die Bedeutung der einzelnen Eigenschaften, wird die technische Zuverlässigkeit der Plattform als weitaus wichtiger bewertet als ausgefallene Funktionalitäten und Dienste. Die Technik der VC-Plattform ist meist nicht der limitierende Faktor, sondern die organisatorische Umsetzung eines Dienstes ist meist ausschlaggebend für dessen Erfolg. Dies zeig sich beispielsweise am Dienst „Fragen an den Experten“: Hier steigen Nutzung und Nutzen des Dienstes signifikant durch schnelle Antwortzeiten sowie qualitativ hochwertige, aber laienverständliche Antworten der Experten. Insgesamt verdeutlichen die Ergebnisse der Evaluation die Notwendigkeit qualitativ hochwertigen Inhalts, der insbesondere als Einstieg für potenzielle Mitglieder Nutzen stiftet.

Als wichtiger Erfolgsfaktor beim Aufbau und Betrieb der VC hat sich das Communitymanagement erwiesen, das sich um Administration und Moderation der VC kümmert und bei noch nicht Erreichen der kritischen Masse an Nutzern für Aktivität und Attraktivität der entstehenden VC sorgen muss.

Weiterhin konnte eine Verlagerung der Informations- und Interaktionsbedarfe der Nutzer empirisch nachgewiesen werden, die mit der Zugehörigkeitsdauer zur Gemeinschaft korreliert ist. Während am Anfang der Mitgliedschaft die Teilnehmer vorrangig Interesse an „harten“ Informationen haben, steigen der Wunsch nach Interaktion und vor allem auch die Wertschätzung derselben bei aktiven Mitgliedern über die Zeit hinweg.

Zusammenfassend kann nach mehrmaligem Durchlaufen des CB\&CM-Vorgehens über insgesamt mehr als zwei Jahre festgehalten werden: Community-Engineering ist möglich und das CB\&CM-Vorgehen ist hierfür ein empirisch nachweislich erfolgreicher Ansatz. Die Analysephase verdeutlicht die Relevanz der Berücksichtigung von Domänenbesonderheiten. Zielgruppen- und mediengerechte Aufbereitung von Gesundheitsinformationen, Usability und Barrierefreiheit des Angebots sowie klare Strukturen bei Oberflächen und Funktionalitäten und vertrauensunterstützende Komponenten sind zentrale Erfolgsfaktoren in der Designphase. Die Einführungs- und Betriebsphase veranschaulicht die zentrale Rolle von Stabilität und Performanz der technischen Plattform, niederschwelligen Möglichkeiten des Erstkontakts mit der VC und der zentralen Rolle des Communitymanagements. Controlling und Evolution zeigen, dass Technik meist nicht der limitierende Faktor für die Akzeptanz eines Communitydiensts ist, sondern dessen organisatorische Umsetzung und dass eine kontinuierliche Weiterentwicklung der $\mathrm{VC}$ auf technischer und organisatorischer Ebene für den dauerhaft erfolgreichen Betrieb zentral ist.

\section{Konsequenzen für die Forschung}

Das Fallbeispiel zeigt, dass erfolgreiches Community-Engineering möglich ist und dass durch VCs die Lebenssituation von Patienten verbessert werden kann. Außerdem ergeben sich folgende Implikationen:

Gesellschaftlich: Ein IT-Pilotierungsforschungsvorhaben im Gesundheitsumfeld kann bereits in der Erprobungsphase die realweltliche Situation von Patienten verbessern. Um mehr gesellschaftlich relevante Ergebnisse zu erzielen gilt es in der Forschung nach weiteren Einsatzbereichen zur Verbesserung der Lebenssituation von $\mathrm{Pa}$ tienten durch bedarfsgerechte IT-Lösungen zu suchen und diese mit geeigneten Methoden, Modellen und Werkzeugen im realen Anwendungszusammenhang zu gestalten. Aktuelle Möglichkeiten hierfür bieten bspw. die elektronische Gesundheitskarte, digitale Assistenzsysteme und ubiquitäre Patienteninformationssysteme.

Sozio-ökonomisch: Es besteht Forschungsbedarf bei der Analyse der Auswirkungen sozialer Interaktionen in VCs auf bspw. das soziale Netzwerk der Mitglieder. Ebenso viel versprechend erscheint die Untersuchung des genauen Nutzens insbesondere gesundheitsbezogener VCs, bspw. in Form der Messung des Einflusses auf die empfundene Lebensqualität und damit mittelbar auf Kriterien wie Lebenserwartung und Behandlungskosten. Weitere offene Fragen ergeben sich im Kontext unterschiedlicher Designmaßnahmen von VCs im Gesundheitswesen. So ist das Verhältnis zwischen einzelnen Einflussgrößen (bspw. vertrauensbildenden Maßnahmen, Stabilität der Plattform, Erweiterung um Funktionalitäten) und dem Erfolg eines Angebotes weder kausal noch quantitativ untersucht worden. Auch sind Rolle und Aufgaben des Communitymanagements in unterschiedlichen Domänen weitgehend unerforscht.

Technisch: Aus technischer Perspektive ergeben sich Chancen und Herausforderungen für die Forschung zu VCs. So sind ubiquitäre Zugänge $\mathrm{zu}$ VCs durch mobile Endgeräte ebenso denkbar wie neue (mobile bzw. kontextsensitive) Dienste für VCs (bspw. ein mobiler Notrufdienst für Krebspatienten mit sofortiger Lokalisierung oder ein mobiler Medikamentenreminder-Dienst, deren Rolle und Nutzen für VCs für Patienten noch nicht abgeschätzt werden können). Außerdem fehlen Arbeiten zu sinnvollen Unterstützungstools für das effiziente und effektive Communitymanagement (insb. bei einer Vielzahl betriebener VCs).

Methodisch: Eine Weiterentwicklung der Pilotierung als Methode der Wirtschaftsinformatik ist notwendig, um für ähnliche Forschungssituationen über ein stärker erprobtes, valides und reliables Forschungsvorgehen $\mathrm{zu}$ verfügen. Die vorgestellte Arbeit kombiniert bestehende Ansätze der Wirtschaftsinformatik zur Pilotierung von IT-basierten Innovationen und wendet diese auf die spezifische Situation von Patienten an, dies kann aber nur ein erster Schritt auf dem Weg der Methodenentwicklung sein.

Theoretisch: Die Pilotierung vermittelt ein reiches Verständnis von interdependenten Einflussgrößen auf Virtuelle Gemeinschaften im Allgemeinen und liefert einen Beitrag zum Verständnis des CommunityBuildings. Auf dieser Basis sind nun Art, Stärke und Richtung der Wechselwirkungen zwischen diesen Größen zu identifizieren um ein darauf abgestimmtes verbessertes Community-Engineering mit verfeinerten Vorgehensweisen zu ermöglichen. Die hier explorativ gewonnenen Ergebnisse können mit VCs aus anderen Lebensbereichen oder anderen Arten von VCs verglichen werden, um die domänenübergreifende Belastbarkeit der Ergebnisse zu erhöhen. Auf Basis dieser Arbeit kann ein quantitativ überprüfbares Modell zum Aufbau und Betrieb von VCs erstellt und so die Theorieentwicklung zu VCs vorangetrieben werden.

Das Community-Engineering muss eine Integration bereits existierender Erkenntnisse und Ansätze aus unterschiedlichen Wissenschaftsdisziplinen leisten. Diese Integrationsperspektive anzubieten und diese Weiterentwicklungsleistung $\mathrm{zu}$ erbringen ist eine Chance für die Wirtschaftsinformatik.

\section{Anmerkungen}

1 Synonym werden in der Literatur die Begriffe „Virtuelle Gemeinschaften“, „Virtual Communities“ und „Online Communities" verwandt. 
${ }^{2}$ Dieser Beitrag ist im Rahmen des Verbundvorhabens COSMOS (Community Online Services and Mobile Solutions) entstanden. Das Projekt wurde vom Bundesministerium für Bildung und Forschung, FKZ 01 HW 0107-0110 gefördert. Weitergehende Informationen unter http://www.cosmos-community.de.

\section{Literatur}

[AWCR04] Alpers, G.; Winzelberg, A.; Classen, C.; Roberts, H.; Dev, P.; Koopman, C.; Taylor, B.: Evaluation of computerized text analysis in an Internet breast cancer support group. In: Computers in Human Behavior 21 (2005) 2, S. $361-$ 376

[ArLK03] Arnold, Y.; Leimeister, J. M.; Krcmar, H.: COPEP: A Development Process Model for a Community Platform for Cancer Patients. In: Proceedings of the XIth European Conference on Information Systems (ECIS), 2003, Neapel.

[AsTa00] Ashry, N. Y.; Taylor, W. A.: Requirements Analysis as Innovation Diffusion: A Proposed Requirements Analysis Strategy for the Development of an Integrated Hospital Information Support System. In: Proceedings of the 33rd Hawai'i International Conference on System Sciences (HICSS 33), 2000, Maui, Hawaii.

[BiDe96] Bilodeau, B. A.; Degner, L. F.: Information needs, sources of information, and decisional roles in women with breast cancer. In: Oncology Nursing Forum 23 (1996) 4, S. 691-696.

[BrMW00] Brunold, J.; Merz, H.; Wagner, J.: www.cyber-communities.de - Virtual Communities: Strategie, Umsetzung, Erfolgsfaktoren. Verlag Moderne Industrie, Landsberg/Lech 2000.

[BuHa00] Bughin, J.; Hagel III, J.: The Operational Performance of Virtual Communities Towards a Successful Business Model? In: Electronic Markets 10 (2000) 4, S. 237-243.

[Coth00] Cothrel, J.: Measuring the success of an online community. In: Strategy \& Leadership 28 (2000) 28, S. 17-21.

[DKLK01] Daum, M.; Klein, A.; Leimeister, J. M.; Krcmar, H.: Webbasierte Informations- und Interaktionsangebote für Onkologiepatienten Ein Überblick. Arbeitspapier Nr. 109. Universität Hohenheim, Lehrstuhl für Wirtschaftsinformatik, Hohenheim 2001.

[Deut06] Deutsche Krebshilfe: Krebs: Zahlen, Daten, Fakten. http://www.krebshilfe.de/neu/ infoangebot/themen/zahlen-daten-fakten/ zahlen.htm, 2006, Abruf am 2006-09-09.

[DiNG03] Dierks, C.; Nitz, G.; Grau, U.: Gesundheitstelematik und Recht. Frankfurter Schriften, MedizinRecht.de-Verlag, Frankfurt a. M. 2003.

[Figa98] Figallo, C.: Hosting Web Communities: Building Relationships, Increasing Customer Loyalty and Maintaining a Competitive Edge. Wiley Computer Publishing, New York 1998.

[FSDM02] Fogg, B. J.; Soohoo, C.; Danielson, D.; Marable, L.; Stanford, J.; Tauber, E. R.: How Do People Evaluate a Web Site's Credibility? Results from a Large Study. Stanford University, Consumer WebWatch, Sliced Bread Design, LLC. http://www.consumerwebwatch.org/ news/report3_credibilityresearch/ stanfordPTL.pdf, 2002, Abruf am 2006-09-09.

[Forb01] Forbiger, A.: Leben ist, wenn man trotzdem lacht. Heyne, München 2001.

[FoFa03] Fox, S.; Fallows, D.: Internet Health Resources. Pew Internet \& American Life Project, Washington 2003

[FKKT98] Frank, U., Klein, S., Krcmar, H., Teubner, A.: Aktionsforschung in der WI - Einsatzpotentiale und Einsatzprobleme. In: Wirtschaftsinformatik und Wissenschaftstheorie. Grundpositionen und Theoriekerne. Arbeitsberichte des Instituts für Produktion und Industrielles Informationsmanagement Nr. 4., Essen 1998, S. 71-90.

[HaAr97] Hagel III, J.; Armstrong, A.: Net Gain Expanding markets through virtual communities. Harvard Business School Press, Boston 1997.

[HeKH01] Heinzl, A.; König, W.; Hack, J.: Erkenntnisziele der Wirtschaftsinformatik in den nächsten drei und zehn Jahren. In: Wirtschaftsinformatik 43 (2001) 3, S. 223-233.

[Hill01] Hiller, B.: Unkonventionelle Verfahren in der Onkologie: der Informationsbedarf der Anrufer beim Krebsinformationsdienst im Deutschen Krebsforschungszentrum zu Methoden mit bisher unbewiesener Wirksamkeit. Dissertation, Universität Heidelberg 2001

[KTCM01] Kaminski, E.; Thomas, R.J.; Charnley, S.; Mackay, J.: Measuring patients response to received information. In: European Journal of Cancer 37 (2001) Supplement 6, S. 387.

[KeJo01] Kelly, K.; Jones, M.: Groupware and the Social Infrastructure of Communication. In Communications of the ACM 44 (2001) 12 S. 77-79.

[Kim99] Kim, A. J.: Secret Strategies for Successful Online Communities/Community-Building On The Web. Peachpit Press, Berkeley 1999.
[KBCSO3] Klemm, P.; Bunnell, D.; Cullen, M.; Soneji, R.; Gibbons, P.; Holecek, A.: Online cancer support groups: A review of the research literature. In: Computer, Informatics, Nursing 21 (2003) 3, S. 136-142.

[KoSm96] Kollock, P.; Smith, M. A.: Managing the Virtual Commons: Cooperation and Conflict in Computer Communities. In: Herring, S. C.; Benjamins, J. (Hrsg.): Computer Mediated Communication: Linguistic, Social and Cross-cultural Perspectives. Amsterdam 1996.

[KöHP95] König, W.; Heinzl, A.; v. Poblotzki, A.: Die zentralen Forschungsgegenstände der Wirtschaftsinformatik in den nächsten 10 Jahren. In: Wirtschaftsinformatik 37 (1995) 6, S. 558-569.

[KRSB05] Krcmar, H.; Reichwald, R.; Schlichter, J.; Baumgarten, U.: Community Services: Healthcare. Josef Eul Verlag, Lohmar 2005.

[LeSc01] Lechner, U.; Schmid, B. F.: Communities - Business Models and System Architectures: The Blueprint of MP3.com, Napster and Gnutella Revisited. In: Proceedings of the 34th Hawai'i International Conference on System Sciences (HICSS 34), 2001, Big Island, Hawaii.

[Leim05] Leimeister, J. M.: Virtuelle Communities für Patienten - Bedarfsgerechte Entwicklung, Einführung und Betrieb. Deutscher Universitäts-Verlag, Wiesbaden 2005.

[LeEK05] Leimeister, J. M.; Ebner, W.; Krcmar, H.: Design, Implementation and Evaluation of Trust-supporting Components in Virtual Communities for Patients. In: Journal of Management Information Systems (JMIS) 21 (2005) 4, S. 101 135.

[LeKr05] Leimeister, J. M.; Krcmar, H.: Evaluation der Akzeptanz und des Nutzens einer systematisch entwickelten Virtuellen Community für Krebspatienten, In: Proceedings of "Wirtschaftsinformatik 2005”, Physica, Bamberg 2005, S. $1063-1082$.

\section{Community-Engineering - Systematic Design and Operation of Virtual Communities in Healthcare}

Virtual communities offer anytime/anyplace access to information and multi-directional exchange with peers in similar situations. They can be beneficial for members and operators. VCs seem especially useful for chronically ill patients since they have strong information and interaction needs. Astonishingly few VCs can be found throughout the German healthcare system and literature neglects that VC could be created and operated systematically.

This article presents an approach to community engineering that is grounded in literature on VCs and innovation research. It is the basis for piloting a VC for cancer patients in Germany. This approach and its underlying design elements and success factors are evaluated longitudinally considering user acceptance, perceived usefulness and emerging virtual social networks. The results show that successful community engineering is possible and the presented approach offers support for the systematic design, implementation and operation of virtual communities in general and in healthcare in particular.

Keywords: Virtual Community, Community Engineering, Piloting, Patient, Health Information, Success Factors, Innovation, Adoption, Diffusion 
[LeRu92] Levenson, R. W.; Ruef, A. W.: Empathy: A psychological substrate. In: Journal of Personality and Social Psychology 63 (1992) 2, S. 234 246.

[Mada97] Madara, E. J.: The mutual-aid self-help online revolution. In: Social Policy, 27 (1997) 3 S. 20-27.

[Mark02] Markus, U.: Integration der virtuellen Community in das CRM: Konzeption, Rahmenmodell, Realisierung. Joseph Eul Verlag, Lohmar 2002.

[Mayh92] Maybew, D. J.: Principles and guidelines in software user interface design. Prentice Hall, Englewood Cliffs 1992.

[McHH01] McPherson, C.J.; Higginson, I. J.; Hearn, J.: Effective methods of giving information in cancer: a systematic literature review of randomized controlled trials. In: Journal of Public Health Medicine 23 (2001) 3, S. 227-234.

[MiSu99] Mills, M. E.; Sullivan, K.: The importance of information giving for patients newly diagnosed with cancer: a review of the literature. In: Journal of Clinical Nursing 8 (1999) 6, S. 631-642.

[Moel96] Moeller, M. L.: Selbsthilfegruppen: Anleitungen und Hintergründe. Rororo-Sachbuch Psychologie aktiv, Rowohlt, Reinbek bei Hamburg 1996.

[Niel02] Nielsen, J.: Coordinating user interfaces for consistency (Nachdruck von 1989). San Francisco 2002.
[OYOF95] Orlikowski, W.; Yates, J.; Okamura, K.; Fujimoto, M.: Shaping Electronic Communication: The Metastructuring of Technology in Use. In: Organization Science 6 (1995) 4, S. 423-444.

[Pree00] Preece, J.: Online Communities - Designing Usability, Supporting Sociability. John Wiley and Sons, Chichester 2000.

[PrGh01] Preece, J.; Ghozati, K.: Observations and Explorations of Empathy Online. In: Internet and Health Communication: Experience and Expectations. In: Rice, R. R.; Katz, J. E. (Hrsg.): The Internet and Health Communication: Experiences and Expectations. Sage, Thousand Oaks 2001, S. 237-260.

[Rhei93] Rheingold, H.: The Virtual Community Homesteading on the Electronic Frontier. Addison Wesley, Reading, MA 1993.

[Roge95] Rogers, E. M.: Diffusion of Innovations. 4. Auflage, The Free Press, New York 1995.

[ScHE99] Schnell, R.; Hill, P. B.; Esser, E.: Methoden der empirischen Sozialforschung. 6., völlig überarbeitete und erweiterte Auflage, Oldenburg, 1999.

[Schu99] Schubert, P.: Virtuelle Transaktionsgemeinschaften im Electronic Commerce: Management, Marketing und Soziale Umwelt. Electronic Commerce, Josef Eul Verlag, Lohmar 1999.

[Schw00] Schwabe, G.: Telekooperation für den Gemeinderat. Kohlhammer, Stuttgart 2000.

[ScKr00] Schwabe, G.; Krcmar, H.: Piloting a Socio-technical Innovation. In: Proceedings of the 8th European Conference on Information Systems (ECIS 2000), S. 132-139, Wien 2000.

[ScLK06] Schweizer, K.; Leimeister, J. M.; Krcmar, H.: The role of virtual communities for the social network of cancer patients. In Proceedings of the Americas Conference on Information Systems (AMCIS 2006), Acapulco 2006.

[SIMW01] Slywotzky, A. J.; Morrison, D. J.; Weber, K.: Get digital! Neue Wege zur Gewinnerzielung durch Digitalisierung. Verlag Moderne Industrie, Landsberg/Lech 2001.

[SmKo99] Smith, M. A.; Kollock, P.: Communities in Cyberspace Routledge, London 1999.

[Spol01] Spolsky, J.: User interface design for programmers. Apress, Berkeley 2001.

[Szyp71] Szyperski, N.: Zur wissensprogrammatischen und forschungsstrategischen Orientierung der Betriebswirtschaft. In: Zeitschrift für Betriebswirtschaft 23 (1971), S. 261-282.

[WEBf06] WEBforALL: Die Bedeutung des Internets für Behinderte. VbI e. V. - Verein zur beruflichen Integration und Qualifizierung e.V.

http://www.webforall.info/html/deutsch/ bedeutung_des_internet.php, 2006, Abruf am 2006-09-09.

[Witt97] Witte, E.: Feldexperimente als Innovationstest - Die Pilotprojekte zu neuen Medien. In: Zeitschrift für betriebswirtschaftliche Forschung 49 (1997) 5, S. 419-436. 